\title{
Research Of Spectrum Sensing Based On Decode and Forward Retransmission
}

\author{
Yuebin Chen \\ college of information engineering \\ Yunnan university of Nationalities \\ Kunming, China \\ cybuestc@sina.com \\ Qian Wang \\ College of Information Engineering \\ Yunnan University of Nationalities \\ Kunmin, China \\ Xing-sheng Pang \\ t1001530@163.com
}

\author{
College of information engineering \\ Yunnan university of Nationalities \\ Kunming, China \\ pangxingsheng@163.com
}

Tuanwei Tian

College of Information Engineering

Yunnan University of Nationalities

Kunming, China

1007054704@163.com

\begin{abstract}
In spectrum sensing, single point detection due to their own limitations is facing some problems such as hidden terminal, perceived sensitivity of the end, etc. Two nodes cooperative spectrum sensing schemes based on DF (Decode and Forward) protocols were proposed to achieve spatial diversity gains in cognitive radio networks, which were referred to DF-CSS(DF-based Cooperative Spectrum Sensing), respectively. This paper is based on DF retransmission spectrum sensing, and analysis the detection of Rayleigh fading environment program for the theoretical. At last, the advantages of collaborative by comparison the cooperative detection with the single point performance is showed.
\end{abstract}

Keywords-Cognitive radio; Cooperation detection; Decode and Forward; Probability of detection

\section{INTRODUCTION}

Spectrum sensing is a key part in the cognitive radio technology, meanwhile, it is also a challenging task. Generally speaking, the spectrum sensing technology is divided into two parts: a single point detection scheme and cooperative detection scheme. Single point detection is easy to realize, but its performance will reduced with the multipath and shadow fading, and itself has some limitations too[1]. Cooperative detection suffers widespread concern because this detection can overcome the limitations of single point detection. A. Ghasemi and other people propose cooperative detection scheme, which can improve overall system probability of detection. But these schemes do not contain the protocol of retransmission[2]. Cooperative spectrum sensing is proposed by G. Ganesan and Li. firstly which based on AF (Amplify and Forward) retransmission[2][3]. But cooperative spectrum sensing which based on DF(Decode and Froward) retransmission few studies. Cooperative diversity based on diversity techniques, that based on the principles of wireless communication environment, and the different transmission will experience different fading. One same signal is able to generate multiple independent 'copy' signal conduct transmission, namely the formation of a "sub-set", which can be effective against fading[6]. Cooperative diversity is divided into two steps. Firstly, the source node sends a broadcast signal, then the destination node and relay nodes receive the signal, which the relay node accept the signal processing; Secondly, the relay node sends a signal to the destination node, and the destination node to merge signals which receive from two steps through some kind of merger.

The cooperative diversity can be divided into three types: the AF-CSS (AF-based Cooperative Spectrum Sensing), the DF-CSS, and the CC-CSS (CC-based Cooperative Spectrum Sensing) according to the relay node processing the received signal. AF-CSS: the relay node receive the signals then amplify and forward retransmission, that G. Ganesan and Li. A detailed study on this method[2][3]; DF-CSS: the relay node receive signals and carry out the judgment, and then forward out, this paper stresses a detailed discussion of this approach; CC-CSS is relay users try its partners to transmit redundant information.

In this paper, the probability of the detection of cooperative spectrum sensing based on DF retransmission is discussed. We analysis the probability of the detection in two situation: cooperation with the DF protocol and noncooperation, that is, signal point performance. It is shown that the cooperation scheme has a better performance than the signal detection. All the results are supported by mathematical analysis and simulations.

\section{SPECTRUM SENSING BASED ON DECODE AND FORWARD}

\section{A. System Model}

Assuming that the wireless network only has two cognitive users $U_{1}$ and $U_{2}$, shown in Figure 1, P denotes the authorized primary user, and the AP is the access point. Cognitive users $U_{1}$ and $U_{2}$ are going to communicate with the access point AP when the primary user $\mathrm{P}$ is to start using 
band. The cognitive user should be detected as soon as possible and quit the band. In this model, $U_{l}$ is far away from the primary user $\mathrm{P}$, and barely receive the signal of the primary user $\mathrm{P}$, so set $U_{2}$ as the cooperative partner of $U_{1}$ that two cognitive users will collaborate. All of the channel in the model is independent, and the receiver attaches to a zero mean and double-sideband power spectral density for the complex Gaussian white noise of $N_{0}$. Figure 2 is a diagram of time slot

If a signal $X$ is sent, the received signal $Y$ is given by

$$
Y=h X+w
$$

where, $h$ is the fading coefficient, $w$ is the additive Gaussian noise.

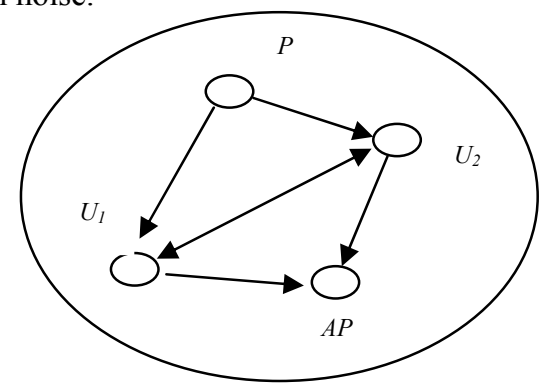

Figure 1. SYSTEM MODEL

TABLE I. COOPERATION IN TWO COGNITIVE USERS

\begin{tabular}{|l|l|l|l|}
\hline$U_{1} T X$ & $U_{2}$ Relay & $U_{2} T X$ & $U_{1}$ Relay \\
\hline
\end{tabular}

Figure 2. RELAY PROTOCOL USED

\section{B. Analysis DF-CSS}

According to DF strategy, cooperative user should estimate whether the primary user on the basis of received signal or not, and retransmit the result to its partner.

The specific process is as follows: In the first slot $t_{1}$, the cognitive user $U_{l}$ transmit own information to the access point AP, while $U_{2}$ receive this information too. In the second slot $t_{2}$, the received information is decoded and judged by $U_{2}$, then the judgment be retransmitted to $U_{1}$. Therefore, we can know that the signal received by the cognitive user $U_{2}$ in time slot $t_{1}$ can be expressed as:

$$
y_{2}=\theta h_{p 2}+\alpha h_{12}+\omega
$$

The signal received by the cognitive user $U_{l}$ in the time slot $t_{2}$ can be expressed as:

$$
y_{1}=\theta h_{p 1}+\hat{\theta} h_{12}+\omega
$$

In (2) and (3), $\theta$ denotes the primary user indicator; $\theta=1$ implies presence of the primary user and $\theta=0$ implies its absence. $h_{p i}$ denotes the instantaneous channel gain between the primary and $U_{i}, \mathrm{i}=1,2 . h_{12}$ denotes the instantaneous channel gain between $U_{1}$ and $U_{2}$. We assume that the channels are reciprocal, i.e., $h_{12}=h_{2}$, and $h_{p 1}, h_{p 2}, \omega$ are zero-mean complex Gaussian random variables which are pairwise independent. The random variables $h_{p 1}, h_{p 2}$ and $h_{12}$ have the variances $\sigma_{p 1}^{2}, \sigma_{p 2}^{2}$ and $\sigma_{12}^{2}$ respectively. $\hat{\theta}$ denotes the estimated indicator, and there is one of two results about the judgment: correct judgment $\hat{\theta}=\theta$ and incorrect judgment $\hat{\theta} \neq \theta$. The detection indicator of the cognitive user will judge the primary user though the two values of the variable $\theta$. $\alpha$ denotes the signal sent form $U_{l}$.

$$
\begin{aligned}
& H_{1}: \theta=1 \\
& H_{0}: \theta=0
\end{aligned}
$$

In this paper, we utilize the energy detector to show advantage of the proposed cooperation scheme. The output of the energy detector is expressed as:

$$
T(y)=|y|^{2}
$$

compared with the threshold $\lambda$ which is determined by a prespecified probability of false alarm $\gamma$, we determine that whether the primary user is presence or not.

\section{The Performance Analysis Of COOPERATIVE SPECTRUM SENSING}

\section{A. Detection Probability}

In this section, we suppose that symbol A denotes $U_{2}$ can correctly detect the primary user $P$ and symbol B denotes $U_{l}$ detect user $P$. According to the total probability formula' we can come to the detection probability of the primary user from $U_{1}$ :

$$
P(B)=P(A) P(B / A)+P(\bar{A}) P(B / \bar{A})
$$

$P(A)$ is the detection probability which the cognitive user correctly detect the primary. According to the received signal in the time slot $t_{1}, U_{2}$ judge the existence of primary user :

$$
\begin{aligned}
& T\left(y_{2}\right)=\left|\theta h_{p 2}+\alpha h_{12}+\omega\right|^{2} \\
& \quad=\theta^{2}\left|h_{p 2}\right|^{2}+\alpha^{2}\left|h_{12}\right|^{2}+N_{0}
\end{aligned}
$$

Since $h_{p 2}$ and $h_{12}$ are complex Gaussian and pairwise independent, it is obvious that $\left|h_{p 2}\right|^{2}$ and $\left|h_{12}\right|^{2}$ are exponential and pairwise independent. Hence, we can get

$$
\begin{aligned}
& P(A)=P\left\{T\left(y_{2}\right) \geq \lambda \mid H_{1}\right\} \\
& =P\left\{\left(\theta^{2}\left|h_{p 2}\right|^{2}+\alpha^{2}\left|h_{12}\right|^{2}\right) \geq \lambda-N_{0} \mid H_{1}\right\} \\
& =\frac{\sigma_{p 2}^{2}}{\sigma_{p 2}^{2}-\sigma_{12}^{2} \alpha^{2}} \exp \left(-\frac{\lambda-N_{0}}{\sigma_{p 2}^{2}}\right) \\
& \quad+\frac{\sigma_{12}^{2} \alpha^{2}}{\sigma_{12}^{2} \alpha^{2}-\sigma_{p 2}^{2}} \exp \left(-\frac{\lambda-N_{0}}{\sigma_{12}^{2} \alpha^{2}}\right)
\end{aligned}
$$

We seek the appropriate detection threshold and assume the probability of false alarm $\gamma$ :

$$
\begin{aligned}
& \gamma=P\left\{T(y) \geq \lambda \mid H_{0}\right\} \\
= & \exp \left(-\frac{\lambda-N_{0}}{\sigma_{12}^{2} \alpha^{2}}\right)
\end{aligned}
$$

That is

$$
\lambda=N_{0}-\sigma_{12}^{2} \alpha^{2} \ln \gamma
$$

The error detection probability which $U_{2}$ detect $\mathrm{P}$ is: 


$$
P(\bar{A})=1-P(A)
$$

$P(B / A)$, the detection of the user $U_{I}$ while the $U_{2}$ made a correct judgment: $\theta=\hat{\theta}$.

$$
\begin{aligned}
y_{1}=\theta h_{p 1}+\hat{\theta} h_{12}+\omega=\theta h_{p 1}+\theta h_{12}+\omega \\
P(B \mid A)=P\left\{T\left(y_{1}\right) \geq \lambda \mid H_{1}\right\} \\
\left.=P\left\{\left.|| h_{p 1}\right|^{2}+\left|h_{12}\right|^{2}\right) \geq \frac{\lambda-N_{0}}{\theta^{2}}\right\} \\
=\frac{\sigma_{p 1}^{2}}{\sigma_{p 1}^{2}-\sigma_{12}^{2}} \exp \left(-\frac{\lambda-N_{0}}{\sigma_{p 1}^{2}}\right) \\
\quad+\frac{\sigma_{12}^{2}}{\sigma_{12}^{2}-\sigma_{p 1}^{2}} \exp \left(-\frac{\lambda-N_{0}}{\sigma_{12}^{2}}\right)
\end{aligned}
$$

$P(B / \bar{A})$, the detection of the user $U_{1}$ while the $U_{2}$ made a incorrect judgment: $\theta \neq \hat{\theta}$.

$$
\begin{gathered}
y_{1}=\theta h_{p 1}+\hat{\theta} h_{12}+\omega \\
P(B \mid \bar{A})=P\left\{T\left(y_{1}\right) \geq \lambda \mid H_{0}\right\} \\
=P\left\{\left|h_{p 1}\right|^{2} \geq \frac{\lambda-N_{0}}{\hat{\theta}^{2}}\right\} \\
=\exp \left(-\frac{\lambda-N_{0}}{\sigma_{p 1}^{2} \hat{\theta}^{2}}\right)
\end{gathered}
$$

Detection threshold $\lambda$ :

$$
\lambda=N_{0}-\sigma_{12}^{2} \hat{\theta}^{2} \ln \gamma
$$

In summary, we get the detection probability of the $U_{1}$ for cooperative spectrum sensing as (6).

When there is no cooperation between $U_{1}$ and $U_{2}, h_{12}$ in (1) is zero. In this case, let $P_{n}(B)$ and $P_{n}(A)$ denote the respective detection probabilities. Now we calculate the detection probability of the $U_{l}$ for no-cooperative:

$$
y_{1}=\theta h_{p 1}+\omega
$$

where, the symbols here have the same meaning as before. Since $y_{1}$ is complex Gaussian, it is obvious that $T\left(y_{1}\right)$ is exponential. Then, we can easily get that

$$
\begin{aligned}
P_{n}(B) & =P\left(T\left(y_{1}\right)>\lambda \mid H_{1}\right) \\
& =\exp \left(-\frac{\lambda}{\sigma_{p 1}^{2}+N_{0}}\right)
\end{aligned}
$$

and

$$
\gamma=P\left(T\left(y_{1}\right)>\lambda \mid H_{0}\right)=\exp \left(-\frac{\lambda}{N_{0}}\right)
$$

From (16) and (17), we can get

$$
P_{n}(B)=\gamma^{\frac{1}{S N R_{1}+1}}
$$

In the same way, we can calculate the detection probability of the $U_{2}$ :

$$
P_{n}(A)=\gamma^{\frac{1}{S N R_{2}+1}}
$$

Where $S N R_{i}$ refers to the received signal power to noise ratio at $U_{i}$ from the primary user.

\section{B. Overall Detection Probability of System}

In the model of the system, the primary user is detected by the cognitive user $U_{1}$ or $U_{2}$, and we can decide presence of the primary user.

According to the rule of the "or", the overall detection probability of system is:

Cooperative detection:

$$
P=1-[1-P(A)]\left[1-P_{n}(B)\right]
$$

No-cooperative detection:

$$
P=1-\left[1-P_{n}(A)\right]\left[1-P_{n}(B)\right]
$$

\section{RESULT AND ANALYSIS}

In this section, the result of the probability of the detection versus signal to noise and probability of false of alarm under Rayleigh environment is shown.

Fig.3 shows the detection probability is different between the cooperative detection and the no-cooperative detection under noise uncertainty:- $1 \mathrm{db}$ and $-1.5 \mathrm{db}$. Follow the signal power to noise ratio, we can know that detection probability of cooperation is higher than no-cooperation.

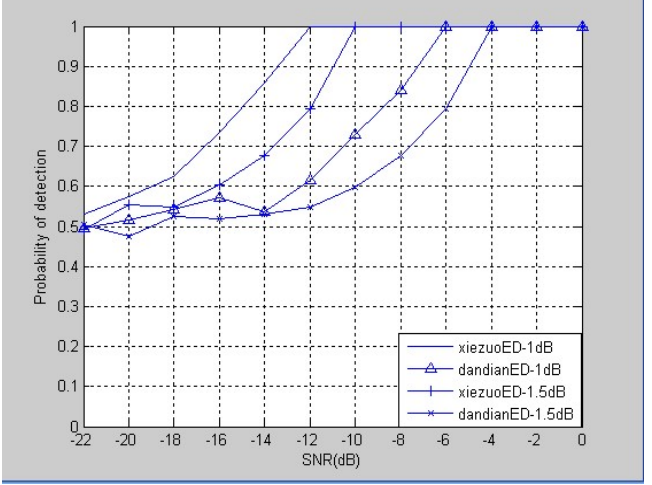

Figure 3. DifFERENT Signal Power to NOISE RATIO

Fig. 4 shows the detection probability is different follow the change of the false alarm $\gamma$. Here the signal to noise is defined -22 and -18 . we can figure out that cooperative detection is better than no-cooperative under the certain signal to noise.

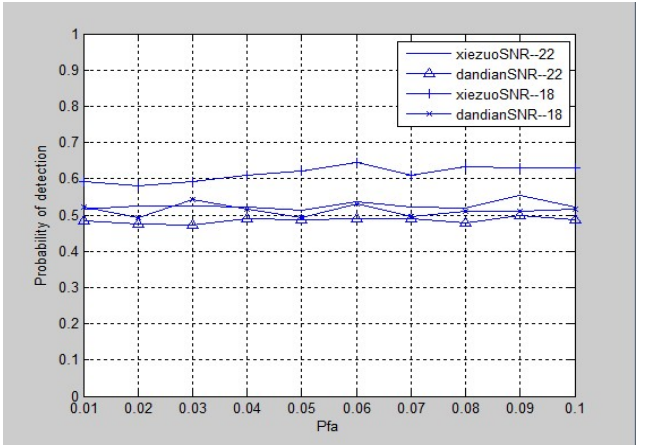

Figure 4. DifFERENT PROBABILITY of FALSE ALARM 


\section{CONCLUSION}

In this section, the probability of detection of the cooperation and noncooperation scheme under Rayleigh environment is discussed. We can know that, DF-CSS can overcome some weakness of the single point detection, so DF-CSS can improve the performance of the cognitive radio. It is shown that, by using DF-CSS protocol, the probability of detection under Rayleigh environment significantly improves at very low SNR range.

\section{ACKNOWLEDGMENT}

This work is supported by the National Natural Science Foundation of China (No. 61261022) and Innovation Team Construction Project of Yunnan University of Nationalities .

\section{REFERENCES}

[1] Amir Ghasemi, Elvino S. Sousa, Collaborative spectrum sensing for opportunistic access in fading environments[C]. Proc IEEE DYSPAN, 2005 .
[2] Ghurumuruhan Ganesan, Ye Li. Cooperative spectrum sensing in cognitive radio, part I: two user networks[J]. IEEE Transactions on Wireless Communications, 2007, 6 (6): 220422213

[3] Ghurumuruhan Ganesan, Ye Li. Cooperative spectrum sensing in cognitive radio, part II :I: Multiuser Networks[J]. IEEE Transaction Wireless Communications, 2007, 6 (6) : 221422222.

[4] Kwang-Cheng Chen, Ramjee Prasad. Cognitive Radio Networks[M]. Wiley, 2009.R. Nicole, "Title of paper with only first word capitalized," J. Name Stand. Abbrev., in press.

[5] youping-zhao. Overhead Analysis for Radio Environment Mapenabled Cognitive Radio Networks[C]. Proc IEEE Reston, VA, USA . 2007 (08) M. Young, The Technical Writer's Handbook. Mill Valley, CA: University Science, 1989.

[6] Dusit Niyato, Ekran Hossain. Competitive spectrum sharing in cognitive radio networks: a dynamic game approach[J]. IEEE Transactions on Wireless Communications, July 2008.

[7] Poisel, Richard. Modern Communications Jamming Principles and $\begin{array}{llll}\text { Techniques[M]. } & \text { Artech } & \text { House. } & 2003\end{array}$ 